BMJ

Open

Gastroenterology

\title{
Impact of statin treatment on non- invasive tests based predictions of fibrosis in a referral pathway for NAFLD
}

\author{
Mustafa Al-Karaghouli (D , , Sonia Fuentes, ${ }^{2}$ Tracy Davyduke, ${ }^{3}$ Mang Ma, ${ }^{1}$ \\ Juan G Abraldes ${ }^{1}$
}

To cite: Al-Karaghouli M, Fuentes S, Davyduke T, et al. Impact of statin treatment on non-invasive tests based predictions of fibrosis in a referral pathway for NAFLD. BMJ Open Gastro 2022;9:e000798. doi:10.1136/ bmjgast-2021-000798

- Additional supplemental material is published online only. To view, please visit the journal online (http://dx.doi. org/10.1136/bmjgast-2021000798).

TD, MM and JGA contributed equally.

TD, MM and JGA are joint senior authors.

Received 5 0ctober 2021 Accepted 28 November 2021

Check for updates

C Author(s) (or their employer(s)) 2022. Re-use permitted under CC BY-NC. No commercial re-use. See rights and permissions. Published by BMJ.

${ }^{1}$ Division of Gastroenterology (Liver Unit), University of Alberta, Edmonton, Alberta, Canada

${ }^{2}$ Family Medicine, University of Alberta, Edmonton, Alberta, Canada

${ }^{3}$ Hepatology Triage Clinic, Alberta Health Services, Edmonton, Alberta, Canada

Correspondence to Dr Juan G Abraldes; juan.g.abraldes@ualberta.ca

\section{ABSTRACT}

Objective In non-alcoholic fatty liver disease (NAFLD), fibrosis determines the risk of liver complications. Noninvasive tests (NITs) such as FIB-4, NAFLD Fibrosis Score (NFS) and Hepamet, have been proposed as a tool to triage NAFLD patients in primary care (PC). These NITs include $A S T \pm A L T$ in their calculations. Many patients with NAFLD take statins, which can affect AST/ALT, but it is unknown if statin affects NITs fibrosis prediction.

Methods We included 856 patients referred through a standardised pathway from PC with a final diagnosis of NAFLD. 832 had reliable vibration controlled transient elastography (VCTE) measurements. We assessed the effects of statins on the association between NITs and VCTE at different fibrosis thresholds.

Results 129 out of 832 patients were taking a statin and 138 additional patients had indication for a statin. For any given FIB-4 value, patients on a statin had higher probabilities of high VCTE than patients not on a statin. Adjusting for body mass index, diabetes and age almost completely abrogated these differences, suggesting that these were related to patient's profile rather to a specific effect of statins. Negative predictive values (NPVs) of FIB-4 $<1.3$ for VCTE $>8,10,12$ and 16 were, respectively, $89,94,96 \%$ and $100 \%$ in patients on a statin and 92 , $95,98 \%$ and $99 \%$ in patients not on a statin. Statins had similar impact on Hepamet predictions but did not modify NFS predictions.

Conclusion In patients with NAFLD referred from PC, those on statins had higher chances of a high VCTE for a given FIB-4 value, but this had a negligible impact on the NPV of the commonly used FIB-4 threshold $(<1.3)$.

\section{INTRODUCTION}

Non-alcoholic fatty liver disease (NAFLD) has become the most common aetiology of chronic liver disease worldwide with a pooled estimated prevalence of $25 \% .^{1}$ The main determinant of liver morbidity in NAFLD is the presence of advanced fibrosis. ${ }^{2} 3$ Indeed, there is an exponential increase in liver related mortality with increased fibrosis stage whereas in patients with no or early fibrosis, the rate of liver related mortality is minimal. ${ }^{3}$

\section{Summary box}

What is already known about this subject?

- In non-alcoholic fatty liver disease (NAFLD), the degree of fibrosis determines the risk of adverse liver outcomes.

- Serum-based non-invasive test for liver fibrosis are recommended to risk-stratify patients with NAFLD.

- NAFLD patients frequently have indication for a statin.

What are the new findings?

- We show a low impact of statin treatment on the reliability of three two-step risk stratification strategies used in patients with NAFLD.

How might it impact on clinical practice in the foreseeable future?

- Patients on statins can be triaged through the same referral pathways as patients not on statins.

Non-invasive tests (NITs) are currently used to risk stratify patients with NAFLD needing to see a liver specialist. The current recommendation is to use a two-step strategy with an initial, simple test with high negative predictive value (NPV), such as FIB-4 or NAFLD Fibrosis Score (NFS), followed by a second test with more positive predictive value such as vibration controlled transient elastography (VCTE). ${ }^{45}$ FIB-4, requires age, platelet count and AST (aspartate aminotransferase)/ $\sqrt{\text { ALT }}$ (alanine aminotransferase) ratio to calculate the score ${ }^{6}$ and has some theoretical advantages since it can be computed directly from routine laboratory results, ${ }^{7-9}$ whereas NFS includes albumin and additional clinical information (body mass index, BMI and presence of diabetes/glucose intolerance). ${ }^{10}$ The recently proposed Hepamet score requires similar variables. ${ }^{11}$

The prevalence of risk factors for cardiovascular disease is very high in patients with 


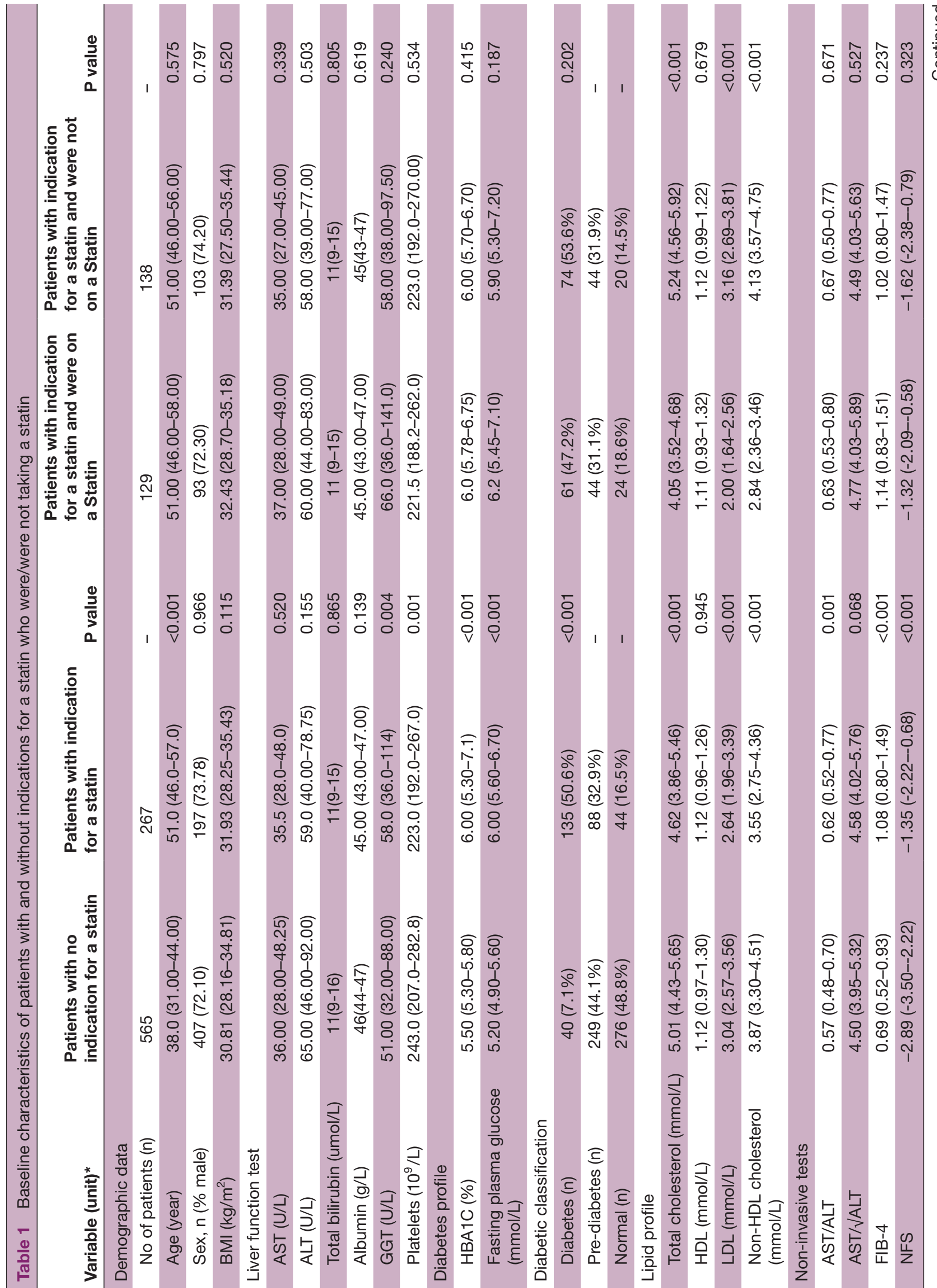


NAFLD. ${ }^{12}{ }^{13}$ Furthermore, NAFLD has been suggested to be an independent risk factor for cardiovascular disease. $^{12} \quad 14 \quad 15$ Hydroxy-methyl-glutaryl-coenzyme A (HMG-CoA) Reductase Inhibitors or statins are the mainstay of primary and secondary prevention of cardiovascular disease. ${ }^{16-18}$ Patients with NAFLD have higher probability of having an indication for statin treatment than patients without NAFLD ( twofold). Previous studies have shown that statins can modify liver transaminases levels, demonstrating both increases or decreases, though the clinical significance of this finding is unclear. ${ }^{19}$ Since most NITs for assessing liver fibrosis are based on either AST or the calculation of some form of AST to ALT ratio, there is the possibility that statins, by modifying transaminases, ${ }^{20-23}$ could modify the performance of these NITs in predicting liver fibrosis. Indeed, a common question among primary care providers (PCPs) is if FIB-4 is still valid in patients on statins. In addition, more advanced NAFLD stages are associated with higher prevalence of metabolic risk factors, especially diabetes and dyslipidaemia, which are the main determinants of statin indication. ${ }^{1724}$ Thus, assessing the impact of statin treatment on non-invasive prediction of liver fibrosis is critical to understand the performance of these NITs as triage methods in NAFLD referral pathways

The aim of this study was to assess if statins modify NITs predictions of fibrosis (determined by VCTE) in patients referred from PC for NAFLD assessment. A secondary aim was to assess the prevalence of statin indication and current use in patients with NAFLD from our referral base.

\section{METHODS}

\section{Study population selection}

We prospectively collected data from asymptomatic patients referred from $\mathrm{PC}$ through a standardised pathway for patients with suspected NAFLD in Edmonton, Canada. Patients were triaged in a nurse-led hepatology triage clinic as previously described. ${ }^{25}$ This study included patients referred from November 2016 to October 2019.

\section{Clinical, laboratory and VCTE assessment}

All patients completed their laboratory tests according to the American College of Gastroenterology to rule out alternative liver diagnosis. ${ }^{26}$ Medical history was obtained by the Registered Nurse who, in this pilot phase of the referral programme, performed VCTE in every patient. A single operator performed all the VCTE tests with a Fibroscan 502 touch (M Probe or XL Probe; Echosens, France) device. We included in this study patients with a final diagnosis of NAFLD after excluding other potential causes of liver disease.

We considered different thresholds for classifying patients as suspected of having advanced fibrosis based on VCTE: $8 \mathrm{kPa}, 10 \mathrm{kPa}, 12 \mathrm{kPa}$ and $16 \mathrm{kPa}$. Each has been proposed to define advanced fibrosis, favouring higher sensitivity and NPV for lower thresholds, or higher 
Statin Status - No Statin - Statin
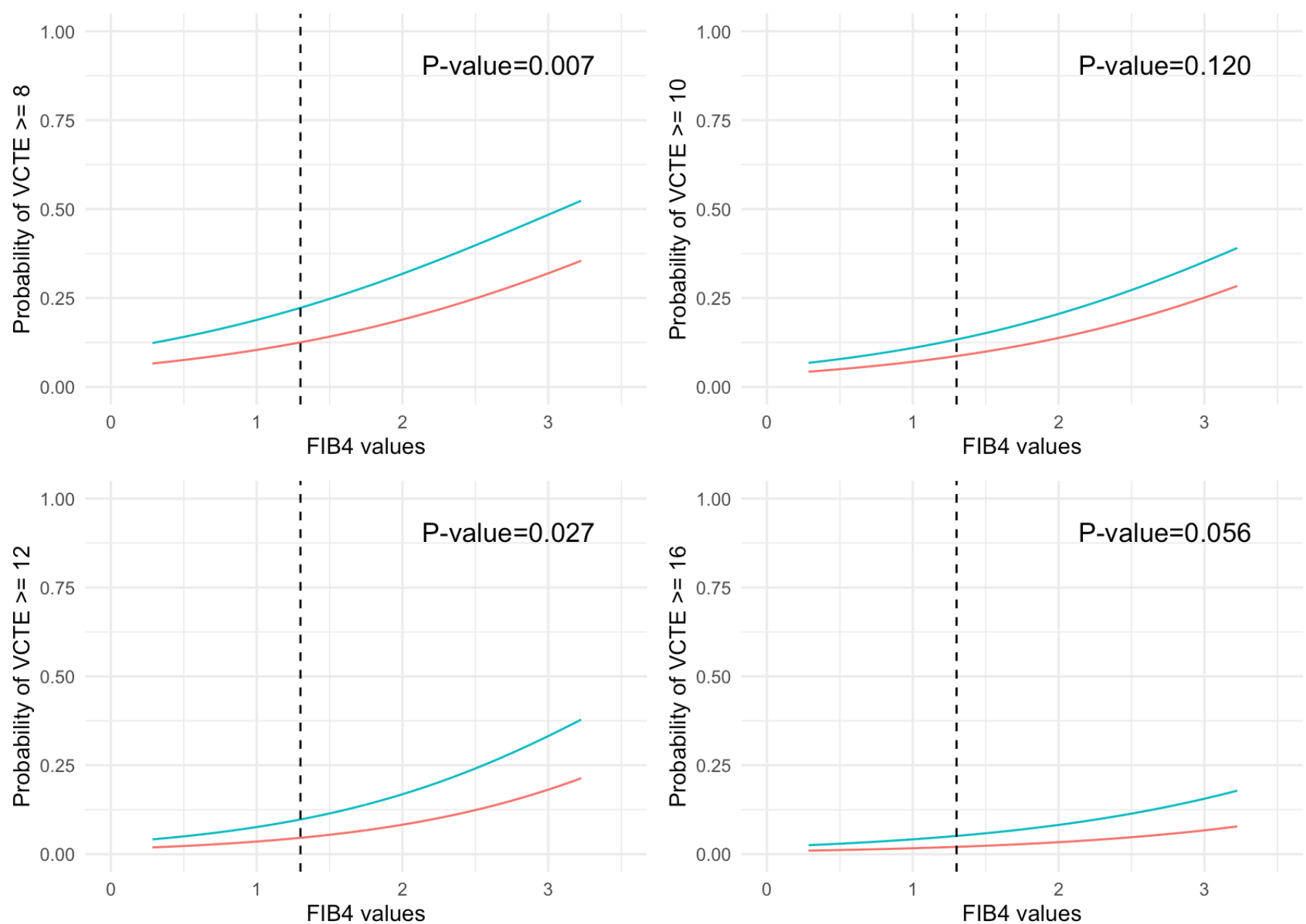

Figure 1 Effect of statin treatment on FIB-4-based prediction of different clinically relevant VCTE thresholds (8, 10, 12 and $16 \mathrm{kPa}$ ). Patients taking a statin had a higher risk of having probabilities of high VCTE than patients not on a statin, but this difference attenuated for higher thresholds. VCTE, vibration controlled transient elastography.

specificity and positive predictive value for higher thresholds. The $16 \mathrm{kPa}$ threshold, in addition, has been recently proposed as an indication to initiate hepatocellular carcinoma (HCC) screening. ${ }^{27-31}$ Unreliable VCTE results were defined as having fewer than 10 valid shots or IQR/ median value greater than $30 \%$ with a VCTE median of $7.1 \mathrm{kPa}$ or higher. ${ }^{32}$ Lab values and clinical data were collected from the local EMR and transferred to a dedicated excel database.

\section{Determination of statin indication}

We used the Canadian Cardiovascular Society guidelines to evaluate how many patients in our sample had an indication for a statin. ${ }^{17}$ Details of how patients were classified as requiring a statin are provided in online supplemental material 1.

\section{Non-invasive tests}

The NITs were used as continuous variables. However, we also tested the previously described thresholds for low-risk for fibrosis to assess NPVs. FIB-4 was calculated as described in Sterling $e t a l^{6}{ }^{6}$ Patients with FIB-4 less than 1.3 were considered as having low risk for advanced fibrosis, while those with higher FIB-4 values were considered to be intermediate/high risk patients requiring further assessment. Hepamet was calculated as described in Ampuero et al with a threshold 0.12 to define low risk. ${ }^{11}$ This threshold is roughly equivalent to the 1.3 FIB- 4 threshold, according to our previous study showing a pointwise risk of VCTE $>8$ of $\sim 12 \%$ in patients with a FIB-4 of $1.3 .^{25}$ NFS was calculated as described in McPherson et al with a threshold of -1.455 defining as low risk. ${ }^{7}$

\section{Statistical analysis}

Median (IQR) was used to describe the numerical variables, whereas absolute and relative frequencies were used to describe the categorical variables. The impact of current statin treatment on the association between NITs and different thresholds of VCTE was modelled using logistic regression (detailed in online supplemental material 2). To further assess whether the effect of statins could be explained by the different clinical 

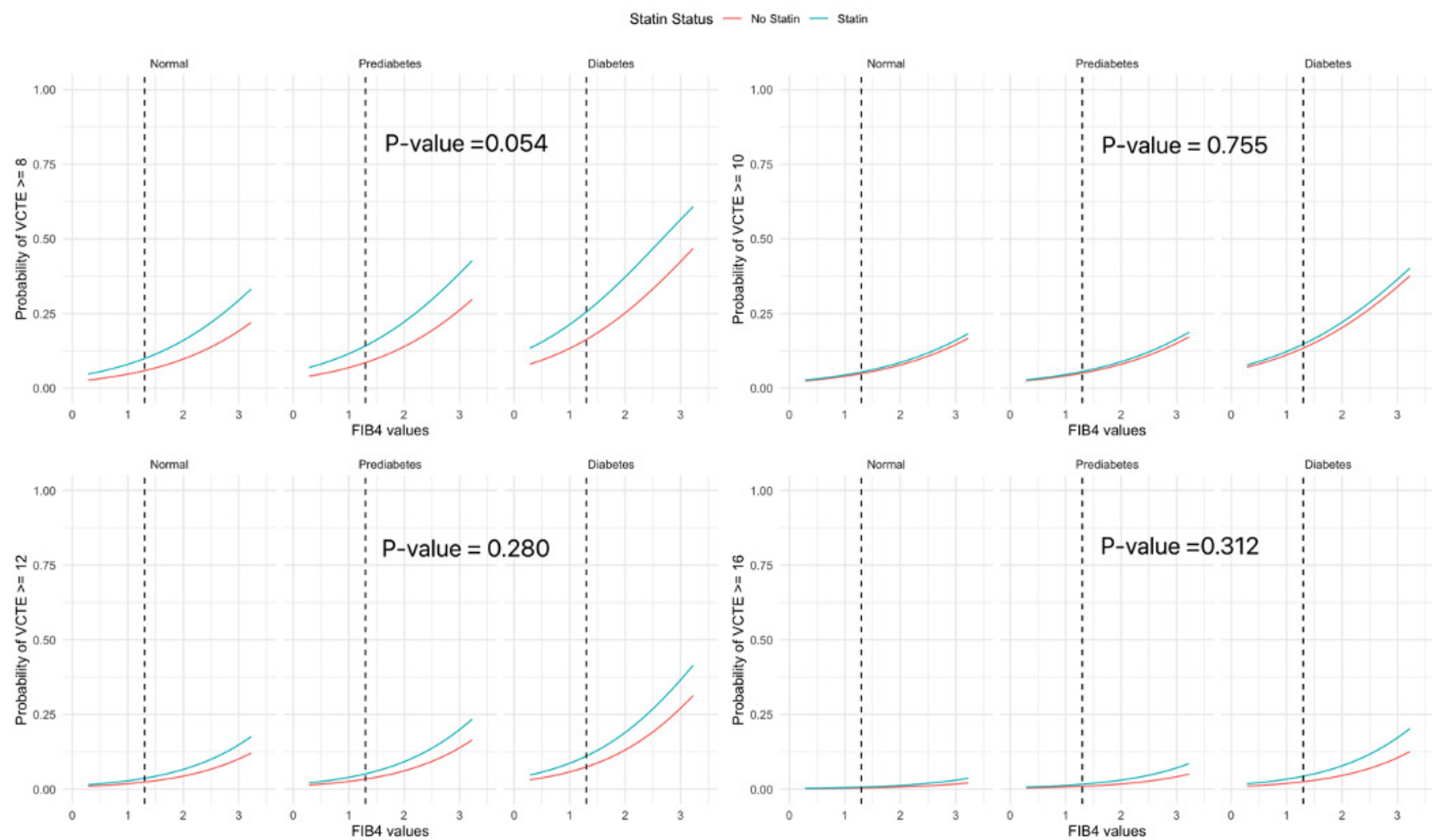

Figure 2 Effect of statin on FIB-4-based prediction of different clinically relevant VCTE thresholds (8, 10, 12 and 16 kPa) after adjusting for age, BMI and diabetes. Adjusting for BMI, diabetes and age almost completely abrogated these differences, suggesting that these were related to patients' profile rather to a specific effect of statins. BMI, body mass index; VCTE, vibration controlled transient elastography.

profile of patients rather than the statin treatment, we conducted additional logistic regression models adjusted by age, diabetes status (classified as normal, pre-diabetes and diabetes), and BMI. To further understand the relation of statin treatment and NITs predictions of fibrosis we used linear regression (detailed in online supplemental material 3). R statistical software (R Foundation for Statistical Computing, Vienna, Austria) was used to conduct the analysis with $\mathrm{rms}^{33}$ and ggplot $2^{34}$ packages.

\section{RESULTS}

\section{Baseline characteristic of the patients}

Of the 934 patients referred to the triage clinic, 856 were classified as having NAFLD. All patients underwent VCTE, and 832 patients had reliable VCTE measurements. Of the 129 patients who were on a statin, 82 were on rosuvastatin, 35 on atorvastatin, 9 on simvastatin and 3 on pravastatin (table 1). One hundred and thirty-eight additional patients had indications for statin treatment according to the Canadian Cardiovascular Society guideline for primary prevention from cardiovascular events but were not on a statin treatment (table 1).

Table 1 shows the baseline characteristics of the patients, according to whether they were on a statin and whether they had an indication for a statin (vs no indication). Online supplemental material 4 shows the characteristics of those on lipophilic statins (atorvastatin or simvastatin) and those on hydrophilic statins (rosuvastatin or pravastatin). Patients with indications for a statin were older and more frequently had diabetes than patients without indication for a statin. There were no differences in liver enzymes or liver function tests between those with indication and those without indication. For every NIT (FIB-4, Hepamet, NFS and VCTE), the median value for those with statin indication was higher than those without indication for a statin. In addition, the prevalence of advanced fibrosis according to VCTE (using different definitions as shown in table 1) was higher in patients with an indication for a statin than the patients without indication for a statin.

Patients with an indication for statin but not on a statin had comparable characteristics to those patients that were actually on a statin, except for their lipid profile (table 1).

\section{Effect of statin use on FIB-4 based predictions of VCTE, with thresholds of $8,10,12$ and $16 \mathrm{kPa}$}

To assess if statins modify FIB-4 based predictions of VCTE, we modelled with logistic regression the association between FIB-4 and VCTE adjusting for statin treatment. For any given value of FIB-4, patients on a statin had higher probabilities of having a VCTE value $>8$ or 
Table 2 The difference between NPV of patients with or without a statin among different NITs

\begin{tabular}{|c|c|c|c|c|}
\hline Statin status & VCTE $>8$ & VCTE > 10 & VCTE >12 & VCTE >16 \\
\hline \multicolumn{5}{|c|}{ FIB-4 $<1.3$ NPVs } \\
\hline Statin & $89 \%$ & $94 \%$ & $96 \%$ & $100 \%$ \\
\hline No statin & $92 \%$ & $95 \%$ & $98 \%$ & $99 \%$ \\
\hline \multicolumn{5}{|c|}{ HEPAMET <0.12 NPVs } \\
\hline Statin & $85 \%$ & $93 \%$ & $94 \%$ & $99 \%$ \\
\hline No statin & $92 \%$ & $95 \%$ & $98 \%$ & $99 \%$ \\
\hline \multicolumn{5}{|c|}{ NFS $<-1.455$ NPVs } \\
\hline Statin & $90 \%$ & $91 \%$ & $95 \%$ & $99 \%$ \\
\hline No statin & $93 \%$ & $96 \%$ & $98 \%$ & $99.6 \%$ \\
\hline
\end{tabular}

NFS, NAFLD Fibrosis Score; NITs, non-invasive tests; NPV, negative predictive value; VCTE, vibration controlled transient elastography.

$10 \mathrm{kPa}$ (figure 1). Of note, there was no significant interaction between FIB-4 and statins, indicating that the effect of statins on FIB-4 predictions was homogeneous across values of FIB- 4 .

We previously showed that age, BMI and diabetes status modify the association between FIB- 4 and VCTE. ${ }^{25}$ In this study the patients on statins were older, had a higher BMI, and more frequently had diabetes (table 1). Thus, to assess if the effect of statins on FIB- 4 based predictions were the results of these differences in patient characteristics, we modelled again the effect of statins, in this case adjusting by BMI, diabetes status and age.

After adjusting for BMI, diabetes and age, the effect of statins was markedly blunted. At VCTE of $8 \mathrm{kPa}$, patients on statins still had higher probabilities of having VCTE $>8 \mathrm{kPa}$, but when higher VCTE thresholds were tested, the difference between patients on statins and not on statins were negligible and non-significant (figure 2).

To further analyse potential confounding factors by indication, we assessed if statin treatment still altered FIB-4 predictions within the subset of patients with indications for statins (that were comparable in every patient characteristic except for the lipid profile). In this subgroup of patients, the effect of statins on FIB-4 predictions behaved similarly as in the adjusted analysis with the full sample, showing some effect only with the VCTE threshold of $8 \mathrm{kPa}$. The difference was progressively attenuated with higher VCTE thresholds and became not significant (online supplemental material 5).

Next, we assessed the impact of statin treatment on the NPVs of FIB- $4<1.3$ for different thresholds of VCTE. FIB- 4 was $<1.3$ in $85 \%$ of the patients not on statins and $64 \%$ of patients on statins. Table 2 shows the NPVs of a FIB-4 $<1.3$ for different VCTE thresholds. Statins had a small impact on the FIB-4 NPVs for detecting a VCTE $<8$, which became negligible for VCTE thresholds of 10, 12 or 16.

Finally, to further understand how statins, BMI, age and diabetes interplay to modify the association between
FIB-4 and VCTE, we conducted a multiple linear regression analysis as detailed in online supplemental material 3 . For a given value of FIB-4, patients on a statin had only a slightly higher mean VCTE value than those not on a statin but the difference was small and non-significant $(p=0.339)$. BMI had a significant and major impact on the FIB-4-based predictions of VCTE. The higher the $\mathrm{BMI}$, the higher the predicted mean VCTE. In the case of diabetes status, only the diagnosis of diabetes but not pre-diabetes had significant effects on predicted mean VCTE as compared with normal patients. The predicted mean VCTE for a given FIB-4 value was higher in diabetes patients than in patients with pre-diabetes or euglycaemia (online supplemental material 3).

\section{Effect of statin use on Hepamet and NFS-based predictions of VCTE}

We additionally tested whether statins impacted Hepamet score and NFS. The effect of statins on the association between Hepamet and VCTE was comparable to that observed with FIB-4. For a given value of Hepamet, patients on statins had higher probabilities of having VCTE $>8$ compared with patients not on statins. The effect of statins on Hepamet became attenuated for higher clinically relevant VCTE values of 10, 12 and $16 \mathrm{kPa}$ (figure 3 ). Ninety-one per cent of patients not on statins and $73 \%$ of the patients on statins were classified as low risk by Hepamet $(<0.12)$. Table 2 shows the NPVs of Hepamet $<0.12$ for different VCTE thresholds for liver fibrosis and these predictions were minimally impacted by statins.

The effect of statins on NFS-based prediction of VCTE was distinct from the effect of FIB- 4 and Hepamet predictions. Indeed, statins did not alter the association between NFS and VCTE even at a low VCTE threshold $(8 \mathrm{kPa})$ (figure 4). However, NFS classified a smaller proportion of patients as low risk ( $83 \%$ on statins and $49 \%$ not on statins) than FIB-4 and Hepamet, while the NPVs were comparable to those of FIB-4 and Hepamet.

\section{DISCUSSION}

In this study, we assessed the potential impact of statin treatment on an NAFLD referral pathway to triage NAFLD patients who need a hepatology referral. We specifically tested the potential modifying effect on statins on three NITs: FIB-4, Hepamet and NFS. In our study, patients who were taking a statin had a higher probability of having VCTE $>8$ for a given value of FIB-4 (though this difference was less marked when using higher VCTE thresholds to define advanced liver fibrosis). We show here that this was mainly related to the higher baseline risk (and hence, pretest probability) of liver fibrosis in patients taking statins. Patients on a statin were older, more frequently had diabetes and had a higher BMI. Second, the effect of statins on the association between Hepamet and VCTE was similar to FIB-4, while it was much less marked for NFS. Overall, statin treatment had 

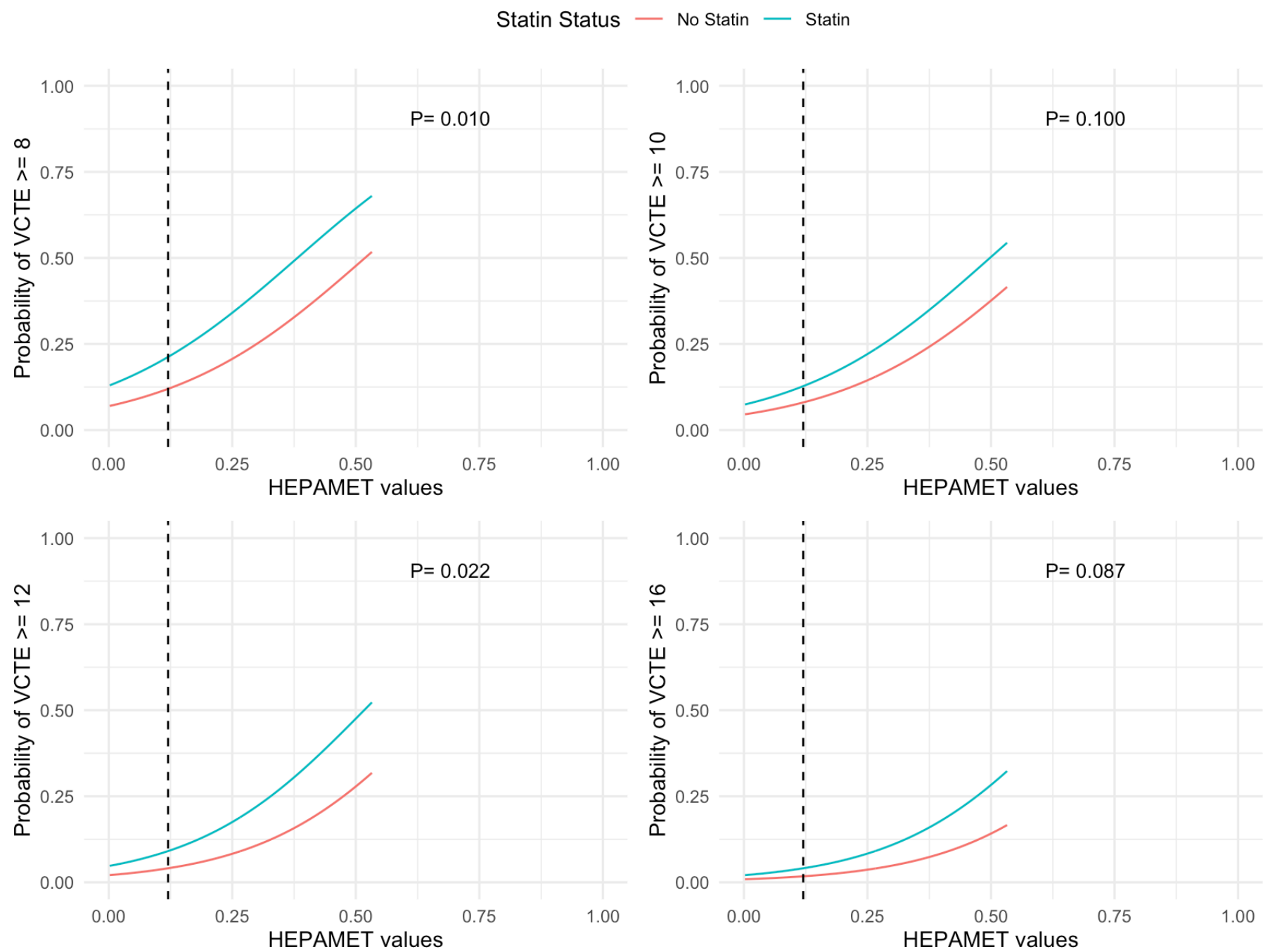

Figure 3 Effects of statin on Hepamet-based prediction of different clinically relevant VCTE thresholds (8, 10, 12 and $16 \mathrm{kPa})$. Patients taking a statin had a higher risk of having probabilities of high VCTE than patients not on a statin, but this difference attenuated for higher thresholds. Vertical dashed line represents the 0.12 threshold. VCTE, vibration controlled transient elastography.

a negligible impact on NPVs of these NITs, and therefore, do not question the value of these NITs as a first step in the referral pathway. Finally, we show that more than half of patients with a clinical indication for a statin were not on a statin at the time of referral.

According to the Canadian Cardiovascular Society, statins are indicated for any patient over the age of 40 with diabetes, which explains why most of our patients with an indication for a statin had more frequently diabetes and were older. ${ }^{17}$ Our adjusted models show that the higher probability of VCTE $>8 \mathrm{kPa}$ for any FIB- 4 value could be mostly explained by the difference in baseline characteristics rather than the effects of the statin itself. When we assessed the effects of statins on FIB-4-based prediction of VCTE in patients with an indication for statins, the prediction difference was not significantly different between those with an indication for a statin and were taking a stain compared with those with an indication for a statin that were not taking a statin. The above analysis provided further supportive evidence that statin treatment itself has a minimal impact on FIB-4 prediction of liver fibrosis, acknowledging the limitation that in this study fibrosis was indirectly assessed by VCTE and not by liver biopsy.

Statins effect on Hepamet-based predictions was comparable to that of FIB-4-based predictions, whereas NFS predictions were minimally altered. This can be explained by the fact that neither Hepamet nor FIB-4 include BMI in their calculation, while this is included in NFS. We have shown before and show again here that BMI has a pronounced effect on NITs based prediction of advanced fibrosis ${ }^{25}$ (online supplemental material 3). Since BMI was different in patients with and without statins, this differential effect between the three NITs is not unexpected. However, in all three NITs, the effect of statins markedly attenuated for higher thresholds of VCTE $(10,12$ and $16 \mathrm{kPa})$. These higher thresholds are probably more in keeping with current trends in the stratification of patients with NAFLD, since the rate of liver related events is negligible in patients with VCTE $<10$ or $12 \mathrm{kPa}^{35}{ }^{36}$ Furthermore, recent guidelines for HCC screening in patients with NAFLD suggest a 

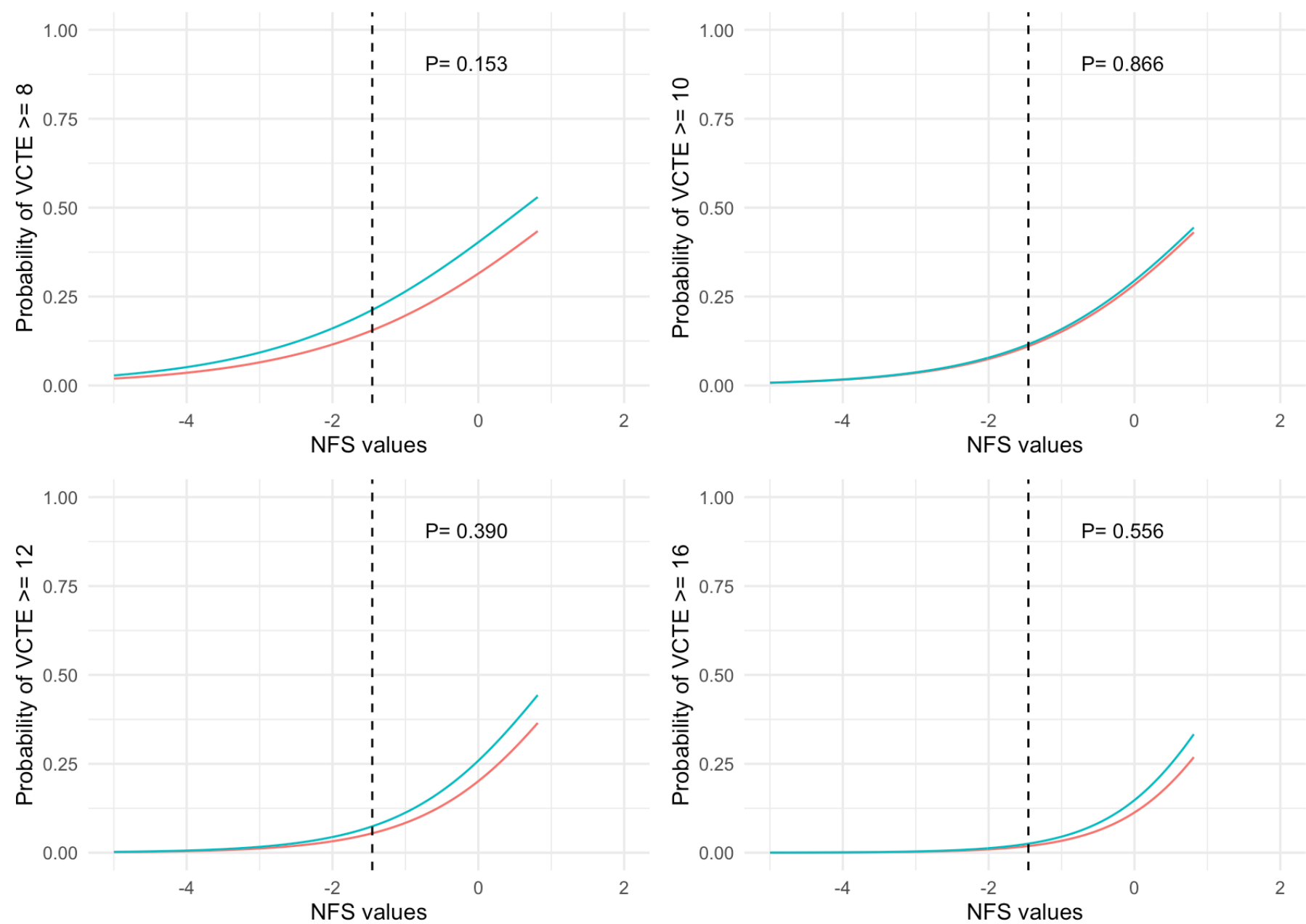

Figure 4 Effect of statins on NFS-based prediction of different clinically relevant VCTE thresholds (8, 10, 12 and 16 kPa). Statin use did not affect NFS predictions of VCTE at any thresholds. The dashed line represents the threshold -1.455. NFS, NAFLD Fibrosis Score; VCTE, vibration controlled transient elastography.

VCTE measurement of $16.1 \mathrm{kPa}$ as the threshold for initiating screening. ${ }^{31}$

Previous studies have shown that statins were under prescribed in patients with NAFLD, with $44 \%-74 \%$ of patients with indication for a statin not receiving a statin. ${ }^{37-39}$ Several explanations might account for this. The most important one could be that PCPs may be concerned about statin liver toxicity in NAFLD patients with baseline serum aminotransferases elevation. ${ }^{40}$ Another explanation could be that, at the time of referral, PCPs could be awaiting hepatologist assessment to decide if statins are contraindicated. In our study, some patients may have been misclassified as having an indication for a statin. Indeed, in a subset of the patients, our definition of statin indication was based on thorough modelling with the data that was available to us (online supplemental material 1), though we show that the impact of this modelling on the calculation of statin indication was minimal. We did not explore the reasons for non-prescription of statins which is a limitation of the study. Moreover, no information about any previous therapy with statins or any potential adverse reactions resulting in statin discontinuation was collected. Finally, the indication of statin in our study was based on the Canadian Cardiovascular Society which has some differences from the American Heart Association guidelines and the European guidelines (that would have resulted in higher numbers of statin indication, and therefore, higher rates of underprescription).$^{24}{ }^{41}$

In conclusion, we have found that statin treatment had only a minor effect on the ability of NITs (FIB-4, Hepamet and NFS) to predict advanced liver fibrosis. This effect was relevant only when a low threshold of VCTE $(8 \mathrm{kPa})$ was used to classify patients with advanced fibrosis, but not with higher thresholds of 10,12 or $16 \mathrm{kPa}$. Our data demonstrate that performance of FIB-4, Hepamet and NFS as a first step in a referral pathway for NAFLD is not significantly affected by statin use. Furthermore, only half of the patients referred through the pathway with an indication for a statin were taking a statin, which calls for 
a further understanding of the factors determining this finding and strategies to optimise cardiovascular prevention, which is still the main cause of morbidity and mortality in NAFLD.

Contributors MA-K: planning the study, collecting and interpreting data, data analysis and drafting the final manuscript. SF and MM: planning the study, interpreting data, drafting the final manuscript and study supervision. TD: planning the study, collecting and interpreting data, drafting the final manuscript and study supervision. JGA: planning the study, interpreting data, data analysis, drafting the final manuscript and study supervision. JGA: is the guarantor of the study

Funding This project was funded in part by an unrestricted grant by Gilead Sciences. Gilead Sciences had no part in the design, analysis, interpretation of the data or in drafting the manuscript. Award/ grant number: IN-CA-989-5661.

Competing interests JGA: Grants/Research Support: Gilead; Speakers Bureau/ Honoraria: Lupin; Consulting Fees: Gilead, Genfit, Intercept, Inventiva, BoehringerIngelheim. MM: Advisory Board: Abbvie, Gilead, Merck, Novartis, Pfizer, Eisai; Funding (Grants): Gilead Science; Research/Clinical Trials: Abbvie, Gilead, Siliagen, Transgene, Pfizer, Exelixis, GSK. MA, SF, TD declare no conflict of interest.

Patient consent for publication Not applicable.

Ethics approval This study involves human participants and was approved by University of Alberta Research Ethics Boards(number Pro00076407). The study was completed as part of a larger quality improvement project that was approved by the University of Alberta Research Ethics Boards.

Provenance and peer review Not commissioned; externally peer reviewed.

Data availability statement Data may be obtained from a third party and are not publicly available. These data are gathered from Alberta Health services (AHS) sources and our current agreement with Alberta Health services does not allow us to share them without an agreement approved by AHS.

Supplemental material This content has been supplied by the author(s). It has not been vetted by BMJ Publishing Group Limited (BMJ) and may not have been peer-reviewed. Any opinions or recommendations discussed are solely those of the author(s) and are not endorsed by BMJ. BMJ disclaims all liability and responsibility arising from any reliance placed on the content. Where the content includes any translated material, BMJ does not warrant the accuracy and reliability of the translations (including but not limited to local regulations, clinical guidelines, terminology, drug names and drug dosages), and is not responsible for any error and/or omissions arising from translation and adaptation or otherwise.

Open access This is an open access article distributed in accordance with the Creative Commons Attribution Non Commercial (CC BY-NC 4.0) license, which permits others to distribute, remix, adapt, build upon this work non-commercially, and license their derivative works on different terms, provided the original work is properly cited, appropriate credit is given, any changes made indicated, and the use is non-commercial. See: http://creativecommons.org/licenses/by-nc/4.0/.

ORCID iD

Mustafa Al-Karaghouli http://orcid.org/0000-0002-0668-6002

\section{REFERENCES}

1 Younossi ZM, Koenig AB, Abdelatif D, et al. Global epidemiology of nonalcoholic fatty liver disease-Meta-analytic assessment of prevalence, incidence, and outcomes. Hepatology 2016;64:73-84.

2 Ekstedt M, Hagström H, Nasr P, et al. Fibrosis stage is the strongest predictor for disease-specific mortality in NAFLD after up to 33 years of follow-up. Hepatology 2015;61:1547-54.

3 Dulai PS, Singh S, Patel J, et al. Increased risk of mortality by fibrosis stage in nonalcoholic fatty liver disease: systematic review and meta-analysis. Hepatology 2017;65:1557-65.

4 Chalasani N, Younossi Z, Lavine JE, et al. The diagnosis and management of nonalcoholic fatty liver disease: practice guidance from the American association for the study of liver diseases. Hepatology 2018;67:328-57.

5 Leoni S, Tovoli F, Napoli L, et al. Current guidelines for the management of non-alcoholic fatty liver disease: a systematic review with comparative analysis. World J Gastroenterol 2018;24:3361-73.

6 Sterling RK, Lissen E, Clumeck N, et al. Development of a simple noninvasive index to predict significant fibrosis in patients with HIV/ HCV coinfection. Hepatology 2006;43:1317-25.
7 McPherson S, Stewart SF, Henderson E, et al. Simple non-invasive fibrosis scoring systems can reliably exclude advanced fibrosis in patients with non-alcoholic fatty liver disease. Gut 2010;59:1265-9.

8 Tsochatzis EA, Newsome PN. Non-alcoholic fatty liver disease and the interface between primary and secondary care. Lancet Gastroenterol Hepatol 2018;3:509-17.

9 Tapper EB, Loomba R. Noninvasive imaging biomarker assessment of liver fibrosis by elastography in NAFLD. Nat Rev Gastroenterol Hepatol 2018;15:274-82.

10 Angulo P, Hui JM, Marchesini G, et al. The NAFLD fibrosis score: a noninvasive system that identifies liver fibrosis in patients with NAFLD. Hepatology 2007;45:846-54.

11 Ampuero J, Pais R, Aller R, et al. Development and validation of hepamet fibrosis scoring system-a simple, noninvasive test to identify patients with nonalcoholic fatty liver disease with advanced fibrosis. Clin Gastroenterol Hepatol 2020;18:216-25.

12 Loomba R, Sanyal AJ. The global NAFLD epidemic. Nat Rev Gastroenterol Hepatol 2013;10:686-90

13 Targher G, Marra F, Marchesini G. Increased risk of cardiovascular disease in non-alcoholic fatty liver disease: causal effect or epiphenomenon? Diabetologia 2008;51:1947-53.

14 Targher G, Day CP, Bonora E. Risk of cardiovascular disease in patients with nonalcoholic fatty liver disease. $N$ Engl J Med 2010;363:1341-50.

15 Martins E, Oliveira A. NAFLD and cardiovascular disease. Porto Biomed J 2018;3:e2

16 Hadjiphilippou S, Ray KK. Cholesterol-lowering agents: Statins -for everyone? Circ Res 2019;124:354-63.

17 Anderson TJ, Grégoire J, Pearson GJ, et al. 2016 Canadian cardiovascular Society guidelines for the management of dyslipidemia for the prevention of cardiovascular disease in the adult. Can J Cardiol 2016;32:1263-82.

18 Stone NJ, Robinson JG, Lichtenstein AH, et al. 2013 ACC/ $\mathrm{AHA}$ guideline on the treatment of blood cholesterol to reduce atherosclerotic cardiovascular risk in adults: a report of the American College of Cardiology/American heart association Task force on practice guidelines. J Am Coll Cardiol 2014;63:2889-934.

19 Sigler MA, Congdon L, Edwards KL. An evidence-based review of statin use in patients with nonalcoholic fatty liver disease. Clin Med Insights Gastroenterol 2018;11:117955221878750.

20 Bader T. Liver tests are irrelevant when prescribing statins. Lancet 2010;376:1882-3.

$21 \mathrm{Kim} \mathrm{H}-\mathrm{S}$, Lee SH, Kim H, et al. Statin-related aminotransferase elevation according to baseline aminotransferases level in real practice in Korea. J Clin Pharm Ther 2016;41:266-72.

22 Nelson A, Torres DM, Morgan AE, et al. A pilot study using simvastatin in the treatment of nonalcoholic steatohepatitis: a randomized placebo-controlled trial. J Clin Gastroenterol 2009;43:990-4.

23 Athyros VG, Tziomalos K, Gossios TD, et al. Safety and efficacy of long-term statin treatment for cardiovascular events in patients with coronary heart disease and abnormal liver tests in the Greek atorvastatin and coronary heart disease evaluation (GREACE) study: a post-hoc analysis. Lancet 2010;376:1916-22.

24 Arnett DK, Blumenthal RS, Albert MA, et al. 2019 ACC/AHA guideline on the primary prevention of cardiovascular disease: executive summary: a report of the American College of Cardiology/ American Heart Association Task Force on Clinical Practice Guidelines. J Am Coll Cardiol 2019;74:1376-414.

25 Davyduke T, Tandon P, Al-Karaghouli M, et al. Impact of Implementing a "FIB-4 First" Strategy on a Pathway for Patients With NAFLD Referred From Primary Care. Hepatol Commun 2019;3:1322-33

26 Kwo PY, Cohen SM, Lim JK. ACG clinical guideline: evaluation of abnormal liver chemistries. Am J Gastroenterol 2017;112:18-35.

27 Cassinotto C, Boursier J, de Lédinghen V, et al. Liver stiffness in nonalcoholic fatty liver disease: a comparison of supersonic shear imaging, FibroScan, and ARFI with liver biopsy. Hepatology 2016:63:1817-27.

28 Caballería L, Pera G, Arteaga I, et al. High prevalence of liver fibrosis among European adults with unknown liver disease: a populationbased study. Clin Gastroenterol Hepatol 2018;16:1138-45.

29 Crespo G, Fernández-Varo G, Mariño Z, et al. ARFI, FibroScan, ELF, and their combinations in the assessment of liver fibrosis: a prospective study. J Hepatol 2012;57:281-7.

30 de LV. Fibrosis assessment in patients with NAFLD. Liver Elastography 2020:123-39.

31 Loomba R, Lim JK, Patton H, et al. AGA clinical practice update on screening and surveillance for hepatocellular carcinoma in patients with nonalcoholic fatty liver disease: expert review. Gastroenterology 2020;158:1822-30 
32 Boursier J, Zarski J-P, de Ledinghen V, et al. Determination of reliability criteria for liver stiffness evaluation by transient elastography. Hepatology 2013;57:1182-91.

33 Harrell Jr FE. Regression modeling strategies: with applications to linear models, logistic and ordinal regression, and survival analysis. Springer, 2015.

34 Wickham H. GGPLOT2: elegant graphics for data analysis 2016. New York: Springer-Verlag, 2016

35 Wong VW-S, Irles M, Wong GL-H, et al. Unified interpretation of liver stiffness measurement by $\mathrm{M}$ and $\mathrm{XL}$ probes in non-alcoholic fatty liver disease. Gut 2019;68:2057-64.

36 Shili-Masmoudi S, Wong GL-H, Hiriart J-B, et al. Liver stiffness measurement predicts long-term survival and complications in nonalcoholic fatty liver disease. Liver Int 2020;40:581-9.
37 Del Ben M, Baratta F, Polimeni L, et al. Under-prescription of statins in patients with non-alcoholic fatty liver disease. Nutr Metab Cardiovasc Dis 2017;27:161-7.

38 Blais $\mathrm{P}$, Lin M, Kramer JR, et al. Statins are underutilized in patients with nonalcoholic fatty liver disease and dyslipidemia. Dig Dis Sci 2016;61:1714-20.

39 Khoo S, Wong VW-S, Goh GB-B, et al. Suboptimal treatment of dyslipidemia in patients with nonalcoholic fatty liver disease. $J$ Gastroenterol Hepatol 2020;35:320-5.

40 Barrios V, Escobar C, Calderón A, et al. Cardiovascular risk profile and risk stratification of the hypertensive population attended by general practitioners and specialists in Spain. The CONTROLRISK study. J Hum Hypertens 2007;21:479-85.

41 Mach F, Baigent C, Catapano AL. ESC/EAS guidelines for the management of dyslipidaemias: lipid modification to reduce cardiovascular risk. Atherosclerosis 2019;2019:140-205. 